\title{
La potencia de las revistas científicas electrónicas para la producción y la circulación del conocimiento en las ciencias sociales y humanas
}

The Power of Electronic Scientific Journals for the Production and Circulation of Knowledge in the Social and Human Sciences

Adela Ruiz

Universidad Nacional de La Plata, Argentina adelaruiz@perio.unlp.edu.ar https://orcid.org/0000-0002-2873-006X

\begin{abstract}
Resumen
La autora reflexiona en este ensayo sobre la importancia del rol que cumplen en el ámbito de las ciencias sociales y humanas las revistas científicas electrónicas a partir de su capacidad de garantizar la efectiva circulación del conocimiento, la calidad científica y editorial de los materiales que ofrecen, el caracter confiable de las fuentes sobre las que se asienta el conocimiento generado, y el avance firme y sostenido del movimiento de acceso abierto a la producción científica y académica.
\end{abstract}

\section{Palabras clave}

Revistas Científicas, Circulación del Conocimiento, Calidad Científica y Editorial, Información Confiable, Acceso Abierto 


\section{Summary}

The author reflects in this essay on the importance of the role that electronic scientific journals play in the field of social and human sciences based on their ability to ensure the effective circulation of knowledge, the scientific and editorial quality of the materials they offer, the reliable character of the sources on which the knowledge generated is based and the firm and sustained advancement of the open access movement to scientific and academic production.

\section{Keywords}

Scientific Journals, Circulation of Knowledge, Scientific and Editorial Quality, Reliable Information, Open Access

En el área científica, la magnitud de la amenaza que el brote de COVID-19 supone para la salud global, ha provocado que la comunicación y la difusión de la información adquieran una velocidad sin precedentes, lo que demuestra, en estos tiempos tan extraños y difíciles que nos toca vivir, el valor indispensable del conocimiento científico para el desarrollo social y económico y para el bienestar de la población.

La publicación de los resultados de las investigaciones constituye no solo una de las actividades más relevantes dentro del quehacer científico sino una responsabilidad para todo aquel que investiga, producto de la contribución que tales comunicaciones cumplen en la democratización del conocimiento (Espinoza Santos, 2010). Y aunque no son el único medio por el cual esta tarea puede materializarse, las revistas especializadas constituyen el soporte más sólido y confiable para la circulación del conocimiento que se genera mediante la investigación científica.

Como sucede con un sinnúmero de los aspectos que podrían analizarse, en el rubro de las publicaciones científicas las diferencias entre las ciencias exactas y naturales y las ciencias sociales o humanas son numerosas y, en algunos casos, muy profundas. No obstante, estas últimas siempre han sido miradas desde la perspectiva de las primeras, lo que obliga a rever de manera constante los criterios que, sin adaptaciones mediante, se trasladan de un ámbito al otro. 
Una de las diferencias que interesa considerar, producto de la importancia que ha adquirido frente a la pandemia global, es la vinculada a la celeridad en la publicación de resultados de investigación. No obstante, por la forma de construcción del conocimiento y por la utilización que se hace del producto de las investigaciones realizadas en un ámbito y en otro, la comparación no es (ni nunca ha sido) viable ni mucho menos recomendable.

En la actualidad, la mirada del mundo está puesta en el trabajo invaluable de quienes podrán responder cuáles son las causas, los modos de propagación, las características, los tratamientos y, confiamos, la vacuna que nos permitirá encontrar una salida médica y sanitaria a la pandemia que nos asola. Pero no menos cierto es que cuando esta coyuntura haya pasado, y comencemos a dejar atrás este (surrealista pero imprescindible) aislamiento social, preventivo y obligatorio, ${ }^{1}$ todo habrá cambiado.

Nuestros vínculos y nuestros modos de encuentro, la manera en la que entendíamos la educación y el rol de las TIC, los modos en el que nos relacionábamos con el medioambiente, la relación que sosteníamos con el consumo, nuestros estilos de alimentación, las formas de representación política, el rol que le atribuíamos a los medios de comunicación, el uso que hacíamos de las redes sociales en nuestra vida cotidiana... Nada será tal como lo conocíamos. Habrá que volver a mirar, a redefinir objetos, a modificar perspectivas, a reformular abordajes metodológicos y será en las revistas científicas del campo de las ciencias sociales y humanas donde los/as investigadores encontrarán el medio capaz de ofrecer la potencia que se requiere para la difusión y la comunicación de estos nuevos conocimientos.

\section{Las revistas como garantía de circulación del conocimiento}

La crisis sanitaria que enfrentamos a nivel mundial no ha hecho más que confirmar algo que, aunque a esta altura del siglo XXI parezca impensado, en las ciencias sociales y humanas (aunque, también, en algunas naturales) continúa siendo motivo de discusión: las revistas científicas son electrónicas o no son científicas.

En las ciencias que interesan para esta reflexión, la adopción de los formatos electrónicos para las revistas científicas no es novedosa, pero tampoco es una realidad generalizada. Se trata de un proceso que, con diferentes niveles de aceptación -los cuales fluctúan acorde a la naturaleza de las disciplinas, las dinámicas de funcionamiento interno de las instituciones y las 
prácticas científicas de las comunidades académicas-, se inició hace tiempo. Incluso, muchas de las publicaciones actualmente posicionadas como referentes en sus campos o en sus áreas temáticas de alcance gestaron su primera edición en el universo digital.

Claro que no se trata de procesos sencillos, ni para las que migran de formato ni para las que nacen en línea. La cultura de la publicación impresa no es fácil de desandar y supone, en especial para los actores que se formaron en el universo editorial del papel, un aprendizaje constante. Pero la riqueza y la potencia de los cambios que se imponen no admiten soluciones mixtas: las revistas científicas no pueden ser temáticas, es válido (y necesario) que periódicamente ofrezcan dossier sobre asuntos especiales, ${ }^{2}$ pero deben estar predispuestas a conformar tablas de contenidos flexibles a los variados intereses de los/as autores/as que las eligen para comunicar los avances de sus investigaciones; su periodicidad no puede encorsetarlas a publicar cada períodos de tiempo que resultarían inviables para cualquier otro medio electrónico, deben tender como escenario deseable a una frecuencia de publicación continua (rolling pass) que las mantenga activas y presentes en las discusiones que atraviesan sus campos disciplinares de pertenencia; sus convocatorias no deben ser dirigidas 0 personalizadas ni limitarse a períodos de tiempo arbitrarios, deben ser abiertas a la comunidad científica de interés y de carácter permanente, para garantizar la celeridad en la publicación de los resultados de investigación y el dinamismo en la circulación del conocimiento que a partir de ellas se difunde.

Más aun cuando es posible disponer, en forma gratuita, de una plataforma como Open Journal Systems (OJS), ${ }^{3}$ que permite administrar todos los aspectos concernientes a la gestión, la edición y la publicación de revistas en línea. Esta herramienta integral, que cuenta con una comunidad muy activa que garantiza su continuo mejoramiento, permite la lectura, la descarga, la copia, la distribución, la impresión, la búsqueda y el enlace a textos completos sin barreras económicas, legales (siempre acorde a las licencias de uso adoptadas) o técnicas.

Las resistencias, que pueden comprenderse pero que no deberían avalarse, aún persisten, aunque, afortunadamente, cada vez en menor medida. $Y$ lo dicho no invalida que también puedan esgrimirse a favor de este cambio los no poco ciertos argumentos económicos relacionados con el costo de sostener las tiradas impresas, sino de comprender que la adopción del soporte electrónico es el único camino posible para que las revistas cumplan con su función vital dentro del ecosistema científico: comunicar y difundir el conocimiento con una rapidez y un alcance impensados (e imposibles) para cualquier publicación impresa. 


\section{Las revistas como garantía de calidad científica}

En tiempos de aislamiento, la generación y el sostenimiento de los vínculos se torna central. Y las revistas científicas electrónicas constituyen, en este sentido, espacios privilegiados de articulación entre especialistas. Sin duda, existen diferentes recursos por medio de los cuales los/as investigadores/as pueden divulgar sus resultados (tesis, congresos, informes de investigación, memorias); no obstante, la dinámica que distingue el proceso editorial que se lleva a cabo en cualquier publicación especializada no solo las convierte en uno de los mejores medios de difusión de los resultados generados por las actividades de investigación y de producción sino, también, en un motor de contactos y de intercambios que, de otro modo, tal vez nunca se producirían.

Artículos originales y evaluados por pares: he aquí la síntesis de lo que divide las aguas en este particular rubro editorial. Requisitos cuya aplicación en nuestros campos (las ciencias sociales y humanas) es considerablemente más reciente que las revistas que en la actualidad los implementan, pero que para beneficio de estos procesos editoriales han dejado de cuestionarse: los materiales se reciben "si no fueron publicados con anterioridad" y se publican «si atravesaron favorablemente la revisión a cargo de especialistas en el campo».

A falta de sistemas que hayan probado funcionar mejor, y apuntalado por las indicaciones (muchas veces, requerimientos disfrazados de sugerencias) de los principales organismos de evaluación, el segundo de estos pilares se aplica mediante el sistema de revisión por pares (Peer Review) que, por lo general, se adopta en su modalidad de doble ciego (Double-Blind Peer review); esto es, los/as autores/as desconocen la identidad de quienes los/as evalúan y los/as evaluadores/as desconocen a quién/quienes corresponde la autoría del artículo sobre el que realizarán la revisión.

Este proceso, que se enriquece producto de la condición según la cual al menos uno de los miembros del cuerpo evaluador no debe pertenecer a la unidad editora (aunque lo ideal sería que ninguno), supone que, como piso mínimo, cada texto que se ofrece en una revista científica ha estado expuesto a la revisión de, al menos, tres especialistas con competencias similares a las de su autor/a: el/la editor/a responsable y la dupla evaluadora, miembros todos de la comunidad científica de que se trate o versados sobre el tema que se pretende publicar. 
Aunque en las ramas de la ciencia que en la actualidad se enfrentan a la carrera contra el coronavirus los tiempos que insume este proceso están siendo fuertemente cuestionados (Méndez, 2020) y han potenciado el incremento de las prepublicaciones o preprints (versiones previas al proceso de revisión por pares mediante el cual se definirá su publicación formal en una revista científica), la discusión no es directamente trasladable al ámbito de las ciencias sociales y humanas. Aquí los/as investigadores no hacen "descubrimientos» capaces de vedar o de obligar a torcer el rumbo de los trabajos de sus pares, sino que el conocimiento se construye mediante discusiones y aportes frente a los cuales el concepto de inmediatez no ocupa el mismo lugar protagónico.

Referimos, hasta aquí, las etapas relacionadas con la validación científica. Pero en términos de producción editorial el fin de esta instancia marca un nuevo comienzo, del que participa una amplia gama de profesionales que hacen posible lo que resta del proceso: editores/as de contenido, correctores/as y revisores/as de estilo, traductores/as, diseñadores/as, maquetadores/as, bibliotecarios/as e informáticos/as que aportan sus saberes y sus habilidades técnicas para que esta dinámica, que se inicia ante cada nueva contribución, funcione de manera ininterrumpida.

En el caso de las universidades, esta maquinaria editorial se sostiene por el trabajo de los equipos editoriales y por el profesionalismo de los equipos técnicos, se valida por el compromiso de los/as especialistas y se materializa por la determinación de las autoridades institucionales. Por eso mismo, y sin dejar de valorar el aporte de quienes impulsaron y en cada momento dan vida al quehacer cotidiano de las diferentes publicaciones periódicas que conforman el acervo editorial de una universidad, considerar que las revistas «son de quienes las organizaron» (Quinteros, 2019) supone desconocer que todas las iniciativas que surgen en los ámbitos de formación superior forman parte del patrimonio científico y académico de instituciones que se construyen, se sostienen y han ganado su prestigio por el aporte y el esfuerzo mancomunado de todos sus integrantes, bajo la premisa de que los nombres propios son relevantes pero, ante todo, coyunturales. Numerosos y diferentes actores, comprometidos con el intercambio de ideas y guiados por la concepción del conocimiento como bien público, se congregan en un mismo espacio: la revista científica como respaldo y como garantía de la transparencia del proceso editorial y de la calidad de los materiales publicados. 


\section{La revistas como garantía de información confiable}

El volumen de información que ha generado la reciente pandemia de COVID-19 ha llevado a la Organización Mundial de la Salud (2019) a señalar que estamos frente a una «infodemia masiva». Informaciones útiles y datos verdaderos mezclados con informaciones manipuladas y datos falsos que dificultan el acceso a fuentes confiables y fidedignas. En este escenario, el trabajo de los diversos actores que intervienen en los procesos de validación del conocimiento que se comunica y que se difunde mediante las revistas científicas juega un rol fundamental.

Desde sus orígenes, a los artículos científicos se les ha pedido que dialoguen tanto con los textos que los preceden como con aquellos que les resultan contemporáneos. En los otrora tiempos de las publicaciones impresas, conocer los antecedentes que fundaron un campo 0 acceder a las principales discusiones que lo estructuraban, sin duda, resultaba más factible que contar con un estado de situación actualizado sobre los trabajos que sobre similares problemas de investigación u objetos de estudio se desarrollaban en otras latitudes, tanto si se compartían las conclusiones o los enfoques como si disentían con sus avances o sus perspectivas.

En el universo de lo digital, y estandarización de los sistemas de citación mediante (en especial, de las no muy queridas Normas APA), ${ }^{4}$ cada artículo que se publica en una revista científica electrónica se convierte en un sólido puente a lecturas especializadas y confiables, que hasta no hace mucho resultaban inaccesibles. Desde siempre, las citas (en sus diferentes variantes: directas, indirectas o de apoyo) han exigido ser acompañadas de los datos que identifican la referencia de origen; desde no hace tanto (concretamente, desde que la producción circula por canales electrónicos), la composición de tales referencias sumó a su composición la necesidad de un nuevo elemento: su ubicación en la red.

«Disponible en», "Consultado el...», «Recuperado de...», aunque con variaciones en las formas de ser presentado, el hipervínculo pasó de ser un recurso optativo en las publicaciones impresas ( $\mathrm{y}, \sin \mathrm{duda}$, con bastante poco sentido) a convertirse en un requisito indispensable para las revistas electrónicas. Y lo hizo bajo una de las principales improntas que distingue el funcionamiento de estas publicaciones: todo recurso que demuestra su utilidad en poco tiempo es mejorado. Así fue como las interminables URL (del inglés, Uniform Resource Locator) ${ }^{5}$ dieron paso a los identificadores doi (del inglés, digital object identifier), ${ }^{6}$ que no solo son más reducidos (para alivio de diseñadores/as y de maquetadores/as) sino que aportan un factor imprescindible en el mundo digital: la identificación unívoca y persistente. 
Este recurso, que en el caso de la Universidad Nacional de La Plata (UNLP) se emplea desde 2017 para todos los artículos que se publican en las revistas científicas que editan las diversas unidades académicas, ${ }^{7}$ no solo asegura la propiedad intelectual de las obras y garantiza la posibilidad de su acceso permanente (es decir, independientemente de los cambios que puedan sufrir las URL originales), sino que incrementa la visibilidad, el acceso y la citación de los artículos, al tiempo que mejora la comunicación entre bases de datos y sistemas informáticos.

De ocupar las páginas finales de un texto, y de constituir el apartado menos atendido por los autores/as y los/as revisores/as, las referencias pasaron a conformar junto con los tradicionales título, resumen y palabras clave la carta de presentación que define el acceso de los/as lectores/as a un material científico; en muchos casos, incluso, representan el único contenido que los/as lectores/as consultan en un trabajo. Las referencias transmiten por sí solas mucha más información de la que en más de una oportunidad ofrece un texto completo y, una vez más, la vigilancia sobre su precisión, su actualidad y su grado de pertinencia es una tarea que descansa sobre el trabajo de edición que resguardan las revistas científicas.

\section{Las revistas como propulsoras del acceso abierto}

Frente a la producción de conocimiento científico más colaborativa de la que se tenga recuerdo, el acceso abierto al conocimiento y a la evidencia científica desempeña, a nivel regional y mundial, un papel decisivo que se traduce en la apertura (en muchos casos, solo temporal) de la literatura científica tendiente a apoyar la búsqueda de soluciones frente a la crisis sanitaria que enfrenta la humanidad como especie.

Tal situación acentúa, con mayor claridad que en cualquier otro momento, lo postulado por los/as defensores/as y propulsores/as del acceso abierto: la necesidad de implementar (y de financiar) políticas que garanticen el libre acceso a la producción científica y académica en pos de contribuir tanto al intercambio sin restricciones como a la reapropiación social del conocimiento científico global.

En nuestra región, hace apenas diez años, el movimiento de Acceso Abierto en las universidades argentinas nacionales y públicas era más el resultado del trabajo de grupos o de individuos claramente identificables que de políticas institucionales que promovieran su establecimiento (Fushimi \& Banzato, 2010). En la actualidad, no obstante, gracias al trabajo 
colaborativo de diversos actores de la comunicación científica, la región cuenta con una propuesta alternativa a la que ofrecen las grandes corporaciones editoriales: AmeliCA, iniciativa puesta en marcha en 2018 con el objetivo de «construir un sistema de comunicación para las revistas latinoamericanas y del Sur Global, en respuesta a la crisis de sustentabilidad económica, la falta de reconocimiento ante los sistemas vigentes de evaluación de la ciencia y ante la exclusión de la mayor parte de las revistas de la región» (AmeliCA, en línea).

Sostenida de forma cooperativa y centrada en el modelo de publicación sin fines de lucro para conservar la naturaleza académica y abierta de la comunicación científica, esta propuesta, liderada por la Organización de las Naciones Unidas para la Educación, la Ciencia y la Cultura (UNESCO), el Consejo Latinoamericano de Ciencias Sociales (CLACSO) y la Red de Revistas Científicas de América Latina, el Caribe, España y Portugal (Redalyc), cuenta con el impulso de tres prestigiosas universidades latinoamericanas: la Universidad Nacional de La Plata (Argentina), la Universidad Autónoma del Estado de México (México) y la Universidad de Antioquia (Colombia).

El rol desempeñado por la UNLP descansa en un elemento central para el sostenimiento de la actividad editorial científica en el ámbito de las universidades nacionales: el apoyo institucional a los espacios de gestión y de producción de revistas científicas en el marco de la aplicación de un modelo de gestión editorial en acceso abierto que ha probado con creces su sustentabilidad (Banzato \& Rozemblum, 2019).

Pruebas de este compromiso institucional con la producción científica en acceso abierto son los cuatro portales de revistas sostenidos por diversas unidades académicas y por diferentes espacios de la UNLP: el Portal de Revistas de la FAHCE, de la Facultad de Humanidades y Ciencias de la Educación, el Portal de Revistas de Periodismo y Comunicación, de la Facultad de Periodismo y Comunicación Social, el Portal de Revistas de Papel Cosido, de la Facultad de Artes, el Portal de Revistas de Informática, de la Facultad del mismo nombre, y el Portal de revistas PREBI-SEDICI, gestionado por el Servicio de Difusión de la Creación Intelectual, el repositorio institucional de la UNLP.

Con la creación de la Coordinación de Revistas Científicas, dependiente de la Dirección de Visibilización de la Producción Científica y Académica de la UNLP, a este escenario se sumó, en diciembre de 2018, el Portal de Revistas de la Universidad Nacional de La Plata, que reúne 
y que permite acceder al casi centenar de revistas científicas, académicas, de divulgación y culturales que son editadas o coeditadas por los diversos órganos que integran la UNLP, en todas las disciplinas temáticas que esta abarca.

Sin duda, aún queda un largo camino por recorrer y numerosas discusiones por sostener, pero los avances en materia de institucionalidad, de coordinación y de estrategia evidencian que el trabajo cooperativo de todos los actores que participan en los procesos de difusión y de comunicación científica es la vía que permitirá garantizar, en el marco de sólidas y de sostenidas políticas de acceso abierto, que el conocimiento científico, entendido como bien social, se constituya en una herramienta clave para el desarrollo y el bienestar de la población.

\section{Referencias}

AmeliCA. Recuperado de http://amelica.org/index.php/que-es-ameli/\#que-es

Banzato, G. y Rozemblum, C. (2019). Modelo sustentable de gestión editorial en Acceso Abierto en instituciones académicas. Principios y procedimientos. Palabra Clave (La Plata), 8(2), e069. https://doi.org/10.24215/18539912e069

Decreto Nacional 297 (2020). Aislamiento social, preventivo y obligatorio. Recuperado de http://servicios.infoleg.gob.ar/infoleglnternet/anexos/335000-339999/335741/norma.htm

Espinosa Santos, V. (2010). Difusión y divulgación de la investigación científica. Idesia (Arica), 28(3), 5-6. https://dx.doi.org/10.4067/S0718-34292010000300001

Fushimi, M. y Banzato, G. (2010). Las políticas de acceso abierto en las universidades estatales argentinas: un análisis a través de la web. Trabajo presentado en las VI Jornadas de Sociología de la UNLP. Recuperado de http://www.memoria.fahce.unlp.edu.ar/trab eventos/ev.931/ev.931.pdf

La UNLP ya cuenta con identificador DOI (3 de agosto de 2017). Recuperado de https://unlp.edu.ar/investigacion/la unlp puede visibilizar su produccion cientifica editada$\underline{1758}$

Larivière, V., Shu, F. y Sugimoto, C. (2020). El brote de coronavirus (COVID-19) resalta serias deficiencias en la comunicación científica [Publicado originalmente en el LSE Impact Blog en marzo/2020]. Recuperado de https://blog.scielo.org/es/2020/03/12/el-brote-de-coronaviruscovid-19-resalta-serias-deficiencias-en-la-comunicacion-cientifica/\#.XsxXOcB7/hE 
Méndez, J. (22 de abril de 2020). Compartir la ciencia en tiempos de coronavirus, una prueba de fuego para el sistema. Dixit. Recuperado de http://dixitciencia.com/2020/04/22/compartir-laciencia-en-tiempos-de-coronavirus-una-prueba-de-fuego-para-el-sistema/

Ministerio de Salud de la Nación (2020). Aislamiento social, preventivo y obligatorio. Recuperado de https://www.argentina.gob.ar/coronavirus/aislamiento

Organización Mundial de la Salud (2019). Entender la infodemia y la desinformación en la lucha contra la COVID-19. Recuperado de https://iris.paho.org/handle/10665.2/52053

Quinteros, G. O. (2019). Sobre las tensiones en los equipos de las revistas científicas universitarias. Palabra Clave (La Plata), 9(1), e074. https://doi.org/10.24215/18539912e074

\section{Notas}

\footnotetext{
${ }^{1}$ Medida excepcional adoptada por el gobierno nacional argentino, mediante el Decreto 297, luego de que la Organización Mundial de la Salud (OMS), con fecha 11 de marzo de 2020, declarara el brote del nuevo coronavirus como una pandemia (Ministerio de Salud, 2020).

${ }^{2}$ Qué mejor ejemplo que la publicación de esta edición especial de la revista Question que hoy nos convoca y las iniciativas que, en esta misma línea, llevan adelante otras revistas de la institución como Revista Argentina de Estudios de Juventud y Tram[p]as de la comunicación y la cultura.

${ }^{3}$ Lanzada y sostenida por el Public Knowledge Project (PKP), una iniciativa multiuniversitaria que desarrolla software de código abierto (gratuito) y realiza investigaciones para mejorar la calidad y el alcance de la publicación académica.

${ }^{4}$ Normas desarrolladas (y periódicamente actualizadas) por la American Psychological Association (APA) que propone un conjunto de estándares para que se unifique, según criterios internacionales comunes, el modo de presentación de los trabajos científicos y el estilo de empleo y de composición de las referencias bibliográficas.

${ }^{5}$ EI URL, que en español significa Localizador Uniforme de Recurso, fue creado y utilizado por primera vez en 1991. A partir de 1994, este concepto fue absorbido por el de URI (Uniform Resource Identifier), debido a que este último era más general. Sin embargo, URL sigue siendo la designación más usual fuera de los ámbitos especializados.

${ }^{6}$ El doi, que en español significa identificador de objeto digital, es un enlace permanente en forma de código alfanumérico que identifica de forma única un contenido electrónico.

7 Su asignación, que es posible desde la que la UNLP adquirió su membresía en CrossRef, es administrada por la Coordinación de Revistas Científicas de la UNLP, a cargo de la Mag. Cecilia Rozemblum.
} 